\title{
DIFFERENTIAL IDENTITIES
}

\author{
BERNARD BEAUZAMY AND JÉROME DÉGOT
}

\begin{abstract}
We deal here with homogeneous polynomials in many variables and their hypercube representation, introduced in [5]. Associated with this representation there is a norm (Bombieri's norm) and a scalar product. We investigate differential identities connected with this scalar product. As a corollary, we obtain Bombieri's inequality (originally proved in [4]), with significant improvements.

The hypercube representation of a polynomial was elaborated in order to meet the requests of massively parallel computation on the "Connection Machine" at Etablissement Technique Central de l'Armement; we see here once again (after [3] and [5]) the theoretical power of the model.
\end{abstract}

\section{THE HYPERCUBE REPRESENTATION AND BOMBIERI'S NORM}

Let

$$
P\left(x_{1}, \ldots, x_{N}\right)=\sum_{|\alpha|=m} a_{\alpha} x_{1}^{\alpha_{1}} \cdots x_{N}^{\alpha_{N}}
$$

be a homogeneous polynomial in $N$ variables $x_{1}, \ldots, x_{N}$, with complex coefficients and degree $m$. Here, as usual, we write $\alpha=\left(\alpha_{1}, \ldots, \alpha_{N}\right),|\alpha|=$ $\alpha_{1}+\cdots+\alpha_{N}$.

For any $i_{1}, \ldots, i_{m}, 1 \leq i_{1} \leq N, \ldots, 1 \leq i_{m} \leq N$, we define, as in [4],

$$
c_{i_{1}, \ldots, i_{m}}=\frac{1}{m !} \frac{\partial^{m} P}{\partial x_{i_{1}} \cdots \partial x_{i_{m}}},
$$

and by Taylor's formula we have

$$
P\left(x_{1}, \ldots, x_{N}\right)=\sum_{i_{i}, \ldots, i_{m}=1}^{N} c_{i_{1}, \ldots, i_{m}} x_{i_{1}} \cdots x_{i_{m}},
$$

which is called the symmetric form of the polynomial.

For a polynomial $P$ of degree $m$, Bombieri's norm is defined as (see [4])

$$
[P]_{(m)}=\left(\sum_{i_{1}, \ldots, i_{m}=1}^{N}\left|c_{i_{1}, \ldots, i_{m}}\right|^{2}\right)^{1 / 2} .
$$

Received by the editors November 2, 1993 and, in revised form, May 18, 1994; originally communicated to the Proceedings of the AMS by Theodore W. Gamelin.

1991 Mathematics Subject Classification. Primary 05A19, 11C08, 30C10.

Key words and phrases. Polynomials, differential identities.

Supported by the C.N.R.S. (France) and the N.S.F. (U.S.A.), by contracts E.T.C.A./C.R.E.A. no. 20367/91, 20388/92, 20432/93 (Ministry of Defense, France), by research contract EERP-FR 22, Digital Equipment Corporation, and by NATO grant CRG 930760.

(C) 1995 American Mathematical Society 
We usually omit the subscript $(m)$, but it should be clear that the norm depends on the degree of the polynomial.

As explained in [5], both (3) and (4) have a geometric description, by means of the hypercube representation of the polynomial: in the hypercube $[0,1]^{m}$, we define the $N^{m}$ points $M_{i_{1}}, \ldots, i_{m}$ with coordinates $i_{1} / N, \ldots, i_{m} / N(1 \leq$ $\left.i_{1} \leq N, \ldots, 1 \leq i_{m} \leq N\right)$. We now put each coefficient $c_{i_{1}, \ldots, i_{m}}$ onto the corresponding point $M_{i_{1}}, \ldots, i_{m}$; this operation is called representation of the polynomial on the hypercube. Bombieri's norm appears as the canonical $l_{2}$ norm associated with this representation.

If we start with any polynomial $P$ given as in (1), it can be written in many ways in the form

$$
P\left(x_{1}, \ldots, x_{N}\right)=\sum_{i_{1}, \ldots, i_{m}=1}^{N} b_{i_{1}, \ldots, i_{m}} x_{i_{1}} \cdots x_{i_{m}},
$$

but the symmetric representation (3) has a particular property, as the following proposition shows (it was communicated to us by Christian Millour):

Proposition 1. Among all representations of $P$ of the form (5), the symmetric one (3) is the one for which the $l_{2}$-norm is minimal.

Proof. For any $i_{1}, \ldots, i_{m}$, if $\sigma$ is a permutation of $\left\{i_{1}, \ldots, i_{m}\right\}$, we have

$$
\sum_{\sigma} b_{\sigma\left(i_{1}\right), \ldots, \sigma\left(i_{m}\right)}=\sum_{\sigma} c_{\sigma\left(i_{1}\right), \ldots, \sigma\left(i_{m}\right)},
$$

and $c_{\sigma\left(i_{1}\right), \ldots, \sigma\left(i_{m}\right)}=c_{i_{1}, \ldots, i_{m}}$ for any $\sigma$. Therefore, the proposition follows from the observation that, if $\left(a_{i}\right)$ are any complex numbers with fixed sum, $\sum\left|a_{i}\right|^{2}$ is minimal when all the $a_{i}$ 's are equal.

\section{THE ASSOCIATED SCALAR PRODUCT}

Canonically associated with Bombieri's norm []$_{(m)}$, there is a scalar product: if $P, Q$ are two homogeneous polynomials with same degree $m$, written in symmetric form as

$$
\begin{aligned}
& P\left(x_{1}, \ldots, x_{N}\right)=\sum_{i_{1}, \ldots, i_{m}=1}^{N} c_{i_{1}, \ldots, i_{m}} x_{i_{1}} \cdots x_{i_{m}}, \\
& Q\left(x_{1}, \ldots, x_{N}\right)=\sum_{i_{1}, \ldots, i_{m}=1}^{N} d_{i_{1}, \ldots, i_{m}} x_{i_{1}} \cdots x_{i_{m}},
\end{aligned}
$$

then we set

$$
[P, Q]_{(m)}=\sum_{i_{1}, \ldots, i_{m}=1}^{N} c_{i_{1}, \ldots, i_{m}} \bar{d}_{i_{1}, \ldots, i_{m}} .
$$

This scalar product appears already in Bruce Reznick [10], where the following result can be found:

Proposition 2. Let $b=\left(b_{1}, \ldots, b_{N}\right)$ and define $\delta_{b}=b_{1} x_{1}+\cdots+b_{N} x_{N}$. Then, for any homogeneous polynomial $P$ with degree $m$,

$$
P\left(b_{1}, \ldots, b_{N}\right)=\left[P, \delta_{\bar{b}}^{m}\right]
$$


Proof. We just observe that $\delta_{\bar{b}}^{m}$ can be written in symmetric form

$$
\delta_{\frac{m}{b}}\left(x_{1}, \ldots, x_{N}\right)=\sum_{i_{1}, \ldots, i_{m}=1}^{N} \bar{b}_{i_{1}} \cdots \bar{b}_{i_{m}} x_{i_{1}} \cdots x_{i_{m}},
$$

and the result follows. This result justifies the notation " $\delta_{b}$ ", since this polynomial behaves as a Dirac measure for this scalar product.

As we did for the norm, we usually omit the subscript $(m)$ in the notation of the scalar product and write simply $[P, Q]$, but one should remember that $P$ and $Q$ must be of the same degree (or be considered so), and that the scalar product depends on that degree.

We observe that, in order to define the scalar product, only one of the polynomials needs to be written in symmetric form:

Proposition 3. Let $P=\sum_{i_{1}, \ldots, i_{m}} c_{i_{1}}, \ldots, i_{m} x_{i_{1}} \cdots x_{i_{m}}$ be written in symmetric form (3), and let

$$
Q=\sum_{j_{1}, \ldots, j_{m}} d_{j_{1}, \ldots, j_{m}} x_{j_{1}} \cdots x_{j_{m}}
$$

be any homogeneous polynomial of degree $m$ (the $d$ 's need not be invariant under permutation of indices). Then

Proof. Let

$$
[P, Q]=\sum_{i_{1}, \ldots, i_{m}} c_{i_{1}, \ldots, i_{m}} \overline{d_{i_{1}}, \ldots, i_{m}} .
$$

$$
Q=\sum_{j_{1}, \ldots, j_{m}} d_{j_{1}, \ldots, j_{m}}^{\prime} x_{j_{1}} \cdots x_{j_{m}}
$$

be the symmetric form of $Q$. Then

$$
[P, Q]=\sum_{i_{1}, \ldots, i_{m}} c_{i_{1}, \ldots, i_{m}} \overline{d_{i_{1}, \ldots, i_{m}}^{\prime}} .
$$

But

$$
d_{j_{1}, \ldots, j_{m}}^{\prime}=\frac{1}{m !} \sum_{\sigma \in S_{m}} d_{j_{\sigma(1)}, \ldots, j_{\sigma(m)}}
$$

where $S_{m}$ is the group of permutations of $\{1, \ldots, m\}$. Substituting into (7) and taking into account the symmetry of the $c$ 's, we obtain the result.

We now investigate a few special situations:

Proposition 4. Let $P_{1}, \ldots, P_{k}$ be homogeneous polynomials in $N$ variables $x_{1}, \ldots, x_{N}$, with degrees $m_{1}, \ldots, m_{k}$. Let $m=m_{1}+\cdots+m_{k}$, and let $q_{1}, \ldots, q_{m}$ be homogeneous polynomials of degree 1 . Then

$$
\begin{aligned}
& {\left[P_{1} \cdots P_{k}, q_{1} \cdots q_{m}\right]} \\
& \quad=\frac{1}{m !} \sum_{\sigma}\left[P_{1}, q_{\sigma(1)} \cdots q_{\sigma\left(m_{1}\right)}\right] \times \cdots \times\left[P_{k}, q_{\sigma\left(m-m_{k}+1\right)} \cdots q_{\sigma(m)}\right],
\end{aligned}
$$

where $\sigma$ runs over all permutations of $\{1, \ldots, m\}$.

Proof. For $i=1, \ldots, m$, we write

$$
q_{i}=\sum_{j=1}^{N} q_{i j} x_{j}
$$


and we obtain a symmetric representation of $q_{1} \cdots q_{m}$ :

$$
q_{1} \cdots q_{m}=\frac{1}{m !} \sum_{i_{1}, \ldots, i_{m}=1}^{N} \sum_{\sigma} q_{1, i_{\sigma(1)}} \cdots q_{m, i_{\sigma(m)}} x_{i_{1}} \cdots x_{i_{m}} .
$$

This can also be written

$$
q_{1} \cdots q_{m}=\frac{1}{m !} \sum_{i_{1}, \ldots, i_{m}=1}^{N} \sum_{\sigma} q_{\sigma(1), i_{1}} \cdots q_{\sigma(m), i_{m}} x_{i_{1}} \cdots x_{i_{m}} .
$$

Now, we write each $P_{j}$ in the form

$$
P_{j}=\sum_{I_{j}} c_{I_{j}}^{(j)} X_{I_{j}}
$$

where $I_{j}=\left\{i_{m_{j-1}+1}, \ldots, i_{m_{j}}\right\}$, and $X_{I_{j}}$ stands for $x_{i_{m_{j-1}+1}} \cdots x_{i_{m_{j}}}$.

Then a nonsymmetric representation of $P_{1} \cdots P_{k}$ is given by

$$
P_{1} \cdots P_{k}=\sum_{I_{1}, \ldots, I_{k}} c_{I_{1}}^{(1)} \cdots c_{I_{k}}^{(k)} X_{I_{1}} \cdots X_{I_{k}} .
$$

Using Proposition 3, we find that

$$
\begin{aligned}
{\left[P_{1} \cdots\right.} & \left.P_{k}, q_{1} \cdots q_{m}\right]=\frac{1}{m !} \sum_{i_{1}, \ldots, i_{m}} \sum_{\sigma} c_{I_{1}}^{(1)} \cdots c_{I_{k}}^{(k)} \bar{q}_{\sigma(1), i_{1}} \cdots \bar{q}_{\sigma(m), i_{m}} \\
= & \frac{1}{m !} \sum_{\sigma}\left(\sum_{I_{1}} c_{I_{1}}^{(1)} \bar{q}_{\sigma(1), i_{1}} \cdots \bar{q}_{\sigma\left(m_{1}\right), i_{m_{1}}}\right) \\
& \times \cdots \times\left(\sum_{I_{k}} c_{I_{k}}^{(k)} \bar{q}_{\sigma\left(m-m_{k}+1\right), i_{m-m_{k}+1}} \cdots \bar{q}_{\sigma(m), i_{m}}\right) \\
= & \frac{1}{m !} \sum_{\sigma}\left[P_{1}, q_{\sigma(1)} \cdots q_{\sigma\left(m_{1}\right)}\right] \cdots\left[P_{k}, q_{\sigma\left(m-m_{k}+1\right)} \cdots q_{\sigma(m)}\right]
\end{aligned}
$$

which concludes the proof.

This proposition has several corollaries (which of course have direct proofs):

Corollary 5. Let $p_{1}, \ldots, p_{m}, q_{1}, \ldots, q_{m}$ be homogeneous polynomials of degree 1 , with variables $x_{1}, \ldots, x_{N}$. Then

$$
\left[p_{1} \cdots p_{m}, q_{1} \cdots q_{m}\right]=\frac{1}{m !} \sum_{\sigma \in S_{m}}\left[p_{1}, q_{\sigma(1)}\right] \cdots\left[p_{m}, q_{\sigma(m)}\right],
$$

where $\sigma$ runs over the set $S_{m}$ of all permutations of $\{1, \ldots, m\}$.

In the next corollary, we give an expression of the scalar product of two polynomials in one variable $z$, with same degree $m$. This expression uses the zeros of both polynomials:

Corollary 6. Let $P=\left(z-a_{1}\right) \cdots\left(z-a_{m}\right), Q=\left(z-b_{1}\right) \cdots\left(z-b_{m}\right)$. Then

$$
[P, Q]=\frac{1}{m !} \sum_{\sigma \in S_{m}}\left(1+a_{1} \bar{b}_{\sigma(1)}\right) \cdots\left(1+a_{m} \bar{b}_{\sigma(m)}\right),
$$

where $\sigma$ turns over the set $S_{m}$ of all permutations of $\{1, \ldots, m\}$. 
This corollary is an obvious consequence of Corollary 5. We identify the one-variable polynomial $z-a$ with the homogeneous two-variable polynomial $z-a z^{\prime}$.

An expression of $[P, Q]$, using the zeros of $P$ and $Q$, was already given by Y. Legrandgérard [8]. It differs from this one, and is more complicated

\section{THE MULTILINEAR FUNCTIONAL ASSOCIATED WITH A MANY-VARIABLE POLYNOMIAL}

Let $P$ be a homogeneous polynomial in $N$ variables. Then there is a multilinear form $L$ on $\mathbb{C}^{N} \times \cdots \times \mathbb{C}^{N}$, associated to this polynomial by the formula

$$
\begin{aligned}
& L\left(Z^{(1)}, \ldots, Z^{(m)}\right) \\
& \quad=\frac{1}{m !} \frac{1}{2^{m}} \sum_{\varepsilon_{1}, \ldots, \varepsilon_{m}= \pm 1} \varepsilon_{1} \cdots \varepsilon_{m} P\left(\sum_{j=1}^{m} \varepsilon_{j} Z_{1}^{(j)}, \ldots, \sum_{j=1}^{m} \varepsilon_{j} Z_{N}^{(j)}\right),
\end{aligned}
$$

where each $Z^{(j)}$ stands for $\left(Z_{1}^{(j)}, \ldots, Z_{N}^{(j)}\right)$.

The fact that $L$ is indeed multilinear is not a priori clear; it was originally proved by Martin in the $30 \mathrm{~s}$ in a Master's thesis. A proof can be found in S. Dineen [6]. But this fact will become obvious once we establish

Proposition 7. The form $L$ coincides with the multilinear form generated by the hypercube; that is,

$$
L_{1}\left(Z^{(1)}, \ldots, Z^{(m)}\right)=\sum_{i_{1}, \ldots, i_{m}=1}^{N} c_{i_{1}, \ldots, i_{m}} Z_{i_{1}}^{(1)} \cdots Z_{i_{m}}^{(m)} .
$$

Since quite obviously $L_{1}$ is linear with respect to each variable, the same will be true for $L$.

Proof. If in (8) we write $P$ in the symmetric form (3) and substitute, we get

$$
\begin{aligned}
L\left(Z^{(1)}, \ldots, Z^{(m)}\right)= & \frac{1}{m ! 2^{m}} \sum_{\varepsilon_{1}, \ldots, \varepsilon_{m}= \pm 1} \varepsilon_{1} \cdots \varepsilon_{m} \sum_{i_{1}, \ldots, i_{m}=1}^{N} c_{i_{1}, \ldots, i_{m}} \\
& \times\left(\sum_{j=1}^{m} \varepsilon_{j} Z_{i_{1}}^{(j)}\right) \cdots\left(\sum_{j=1}^{m} \varepsilon_{j} Z_{i_{m}}^{(j)}\right) \\
= & \frac{1}{m ! 2^{m}} \sum_{i_{1}, \ldots, i_{m}=1}^{N} c_{i_{1}, \ldots, i_{m}} \sum_{\varepsilon_{1}, \ldots, \varepsilon_{m}= \pm 1} \varepsilon_{1} \cdots \varepsilon_{m} \\
& \times\left(\sum_{j=1}^{m} \varepsilon_{j} Z_{i_{1}}^{(j)}\right) \ldots\left(\sum_{j=1}^{m} \varepsilon_{j} Z_{i_{m}}^{(j)}\right) .
\end{aligned}
$$

Using Rademacher functions (see for instance [2]), this can be written more simply:

$$
L=\frac{1}{m !} \sum_{i_{1}, \ldots, i_{m}=1}^{N} c_{i_{1}, \ldots, i_{m}} \int_{0}^{1} r_{1}(t) \cdots r_{m}(t)\left(\sum_{j=1}^{m} r_{j}(t) Z_{i_{1}}^{(j)}\right) \ldots\left(\sum_{j=1}^{m} r_{j}(t) Z_{i_{m}}^{(j)}\right) d t .
$$


We expand all products and observe that all terms have integral 0 , except those which give $r_{1}^{2}(t) \cdots r_{m}^{2}(t)$; the latter have integral 1 . This way, we obtain

$$
L=\frac{1}{m !} \sum_{u} \sum_{i_{1}, \ldots, i_{m}=1}^{N} c_{i_{1}, \ldots, i_{m}} Z_{i_{1}}^{\left(u_{1}\right)} \cdots Z_{i_{m}}^{\left(u_{m}\right)},
$$

where $u=\left(u_{1}, \ldots, u_{m}\right)$ runs through all permutations of $\{1, \ldots, m\}$. We rewrite

$$
Z_{i_{1}}^{\left(u_{1}\right)} \cdots Z_{i_{m}}^{\left(u_{m}\right)}=Z_{i_{u^{-1}(1)}^{(1)}}^{(1)} \cdots Z_{i_{u^{-1}(m)}^{(m)}}^{(m)},
$$

and use the fact that $c_{i_{u}-1_{(1)}}, \ldots, i_{u^{-1} 1_{(m)}}=c_{i_{1}}, \ldots, i_{m}$, and obtain the result.

Example 8. The polynomial $P\left(x_{1}, x_{2}\right)=x_{1}^{3}-3 x_{1}^{2} x_{2}+x_{2}^{3}$ gives in symmetric form $x_{1}^{3}-\left(x_{1} x_{1} x_{2}+x_{1} x_{2} x_{1}+x_{2} x_{1} x_{1}\right)+x_{2}^{3}$, and the associated trilinear form is:

$$
\begin{aligned}
& L\left(Z^{(1)}, Z^{(2)}, Z^{(3)}\right)=Z_{1}^{(1)} Z_{1}^{(2)} Z_{1}^{(3)} \\
& \quad-\left(Z_{1}^{(1)} Z_{1}^{(2)} Z_{2}^{(3)}+Z_{1}^{(1)} Z_{2}^{(2)} Z_{1}^{(3)}+Z_{2}^{(1)} Z_{1}^{(2)} Z_{1}^{(3)}\right)+Z_{2}^{(1)} Z_{2}^{(2)} Z_{2}^{(3)} .
\end{aligned}
$$

As pointed out to us by Andrew Tonge, the above proposition is known to specialists in multilinear functionals, though we could not find a published proof. Moreover, it does not seem to provide any quantitative improvement of the results on the norm of the multilinear functional obtained originally by Banach and completed by various authors (R. Aron and J. Globevnik [1], L. Harris [7], Y. Sarantopoulos [11], I. Zalduendo [12]).

\section{Differential IDENTITIES}

Let us return to scalar products. The basic observation (already made by Bruce Reznick in [10]) is that multiplication by a variable on one side becomes derivation on the other side. Precisely, we have:

Lemma 9. If $P$ is of degree $m-1$ and $Q$ of degree $m$, then

$$
\left[x_{1} P, Q\right]_{(m)}=\frac{1}{m}\left[P, \frac{\partial Q}{\partial x_{1}}\right]_{(m-1)} .
$$

Proof. Of course, it is enough to prove the formula when $P, Q$ are monomials, say $P=x_{1}^{\alpha_{1}} \cdots x_{N}^{\alpha_{N}}$ (with $\left.|\alpha|=m-1\right), Q=x_{1}^{\beta_{1}} \cdots x_{N}^{\beta_{N}}$ (with $(|\beta|=m)$. Then

$$
\left[x_{1} P, Q\right]_{(m)}=\left[x_{1}^{\alpha_{1}+1} x_{2}^{\alpha_{2}} \cdots x_{N}^{\alpha_{N}}, x_{1}^{\beta_{1}} \cdots x_{N}^{\beta_{N}}\right]_{(m)}
$$

and this is 0 , except if $\beta_{1}=\alpha_{1}+1, \beta_{2}=\alpha_{2}, \ldots, \beta_{N}=\alpha_{N}$, in which case the value is

Similarly,

$$
\left[x_{1}^{\beta_{1}} \cdots x_{N}^{\beta_{N}}\right]_{(m)}^{2}=\frac{\beta_{1} ! \cdots \beta_{N} !}{m !} \text {. }
$$

$$
\left[P, \frac{\partial Q}{\partial x_{1}}\right]_{(m-1)}=\left[x_{1}^{\alpha_{1}} \cdots x_{N}^{\alpha_{N}}, \beta_{1} x_{1}^{\beta_{1}-1} x_{2}^{\beta_{2}} \cdots x_{N}^{\beta_{N}}\right]_{(m-1)}
$$

is 0 , except if $\alpha_{1}=\beta_{1}-1, \alpha_{2}=\beta_{2}, \ldots, \alpha_{N}=\beta_{N}$, in which case the value is

$$
\beta_{1}\left[x_{1}^{\alpha_{1}} \cdots x_{N}^{\alpha_{N}}\right]_{(m-1)}^{2}=\beta_{1} \frac{\alpha_{1} ! \cdots \alpha_{N} !}{(m-1) !}=\frac{\beta_{1} ! \cdots \beta_{N} !}{(m-1) !} ;
$$

the result follows. 
Corollary 10 (Transpositon of a linear factor). Let $P$ be of degree $m-1$ and $Q$ be of degree $m$. Then

$$
\left[\left(\sum a_{j} x_{j}\right) P, Q\right]_{(m)}=\frac{1}{m}\left[P, \sum \bar{a}_{j} \frac{\partial Q}{\partial x_{j}}\right]_{(m-1)} .
$$

If $P$ and $Q$ are one-variable polynomials (with the identification between $\sum_{0}^{m} a_{j} z^{j}$ and $\left.\sum_{0}^{m} a_{j} z^{j} z^{\prime m-j}\right)$, the polynomial $a \partial Q / \partial z+a^{\prime} \partial Q / \partial z^{\prime}$ is the homogeneous version of the polynomial $m Q(z)+(a-z) Q^{\prime}(z)$, which is called the polar derivative of $Q$ at the point $a$ (cf. Marden [9]) and denoted by $Q_{1}(a, z)$. So, for one-variable polynomials, the above formula becomes

$$
[(a z+1) P, Q]_{(m)}=\frac{1}{m}\left[P, Q_{1}(\bar{a}, z)\right]_{(m-1)} .
$$

From Lemma 9 will follow several differential identities. In order to state them, we recall the following definitions, which are standard in P.D.E.

If $P\left(x_{1}, \ldots, x_{N}\right)=\sum a_{\alpha} x_{1}^{\alpha_{1}} \cdots x_{N}^{\alpha_{N}}$ is a polynomial, the associated differential operator is

$$
P\left(D_{1}, \ldots, D_{N}\right)=\sum a_{\alpha} D_{1}^{\alpha_{1}} \cdots D_{N}^{\alpha_{N}}
$$

where $D_{j}$ stands for $\partial / \partial x_{j}$. This operator is usually written simply $P(D)$, with $D=\left(D_{1}, \ldots, D_{N}\right)$. We write simply $P_{i}$ instead of $\partial P / \partial x_{i}$, and more generally $P_{i_{1}, \ldots, i_{k}}$ instead of $\partial^{k} P / \partial x_{i_{1}} \cdots \partial x_{i_{k}}$.

We also define

$$
P^{*}\left(x_{1}, \ldots, x_{N}\right)=\sum_{|\alpha|=m} \overline{a_{\alpha}} x_{1}^{\alpha_{1}} \cdots x_{N}^{\alpha_{N}} .
$$

A simple generalization of Lemma 9, also stated by Bruce Reznick [10], is

Lemma 11. Let $P, Q, R$ be homogeneous polynomials, with $\operatorname{deg} P=p$, $\operatorname{deg} Q=q, \operatorname{deg} R=r$, with $r=p+q$. Then

$$
[P Q, R]_{(r)}=\frac{q !}{r !}\left[Q, P^{*}(D) R\right]_{(q)} .
$$

We can now state the most general form of the differential identities.

Theorem 12. Let $P, Q, R, S$ be four homogeneous polynomials, respectively of degree $p, q, r, s$, with $p+q=r+s$. Then

$$
\begin{aligned}
& {[P Q, R S]_{(p+q)}} \\
& \quad=\frac{1}{(p+q) !} \sum_{k \geq 0} \frac{(q-r+k) !}{k !} \sum_{i_{1}, \ldots, i_{k}=1}^{N}\left[R_{i_{1}, \ldots, i_{k}}^{*}(D) Q, P_{i_{1}, \ldots, i_{k}}^{*}(D) S\right]_{(q-r+k)} .
\end{aligned}
$$

We observe that in this sum the terms are 0

- if $k>p\left(\right.$ since $\left.P_{i_{1}}^{*}, \ldots, i_{k}=0\right)$,

- if $k>r\left(\right.$ since $\left.R_{i_{1}}^{*}, \ldots, i_{k}=0\right)$,

- if $r-k>q\left(\right.$ since $\left.R_{i_{1}}^{*}, \ldots, i_{k}(D) Q=0\right)$, and

- if $r-k>s$ (since $P_{i_{1}}^{*}, \ldots, i_{k}(D) S=0$ ). 
We will give two proofs of the theorem: the first one is longer but more transparent, the second one shorter and less transparent.

First proof. If $P=\sum c_{i_{1}}, \ldots, i_{p} x_{i_{1}} \cdots x_{i_{p}}, R=\sum d_{j_{1}, \ldots, j_{r}} x_{j_{1}} \cdots x_{j_{r}}$, then we have

$$
\begin{aligned}
& {[P Q, R S]=\sum_{i_{1}, \ldots, i_{p}} \sum_{j_{1}, \ldots, j_{r}} c_{i_{1}, \ldots, i_{p}} \bar{d}_{j_{1}, \ldots, j_{r}}\left[x_{i_{1}} \cdots x_{i_{p}} Q, x_{j_{1}} \cdots x_{j_{r}} S\right]_{p+q}} \\
& =\frac{1}{p+q} \sum_{i_{1}, \ldots, i_{p}} \sum_{j_{1}, \ldots, j_{r}} c_{i_{1}, \ldots, i_{p}} \bar{d}_{j_{1}, \ldots, j_{r}}\left[x_{i_{2}} \cdots x_{i_{p}} Q, \frac{\partial}{\partial x_{i_{1}}}\left(x_{j_{1}} \cdots x_{j_{r}} S\right)\right]_{p+q-1},
\end{aligned}
$$

by Lemma 9.

We now need a lemma which describes the exchange between a multiplication and a derivation.

Lemma 13. For all homogeneous polynomials $R, Q$ with same degree,

$$
\sum_{i, j} c_{i, j}\left[R, \frac{\partial}{\partial x_{i}}\left(x_{j} Q\right)\right]=\left(\sum_{j} c_{j, j}\right)[R, Q]+\sum_{i, j} c_{i, j}\left[R, x_{j} \frac{\partial Q}{\partial x_{i}}\right] .
$$

Proof of Lemma 13. We have simply

$$
\begin{aligned}
\sum_{i, j} c_{i, j} & {\left[R, \frac{\partial}{\partial x_{i}}\left(x_{j} Q\right)\right] } \\
= & \sum_{j} \sum_{i \neq j} c_{i, j}\left[R, \frac{\partial}{\partial x_{i}}\left(x_{j} Q\right)\right]+\sum_{j} c_{j, j}\left[R, \frac{\partial}{\partial x_{j}}\left(x_{j} Q\right)\right] \\
= & \sum_{j} \sum_{i \neq j} c_{i, j}\left[R, x_{j} \frac{\partial Q}{\partial x_{i}}\right]+\sum_{j} c_{j, j}\left[R, x_{j} \frac{\partial Q}{\partial x_{j}}+Q\right]
\end{aligned}
$$

and the lemma follows.

We observe that the statement of this lemma is quite similar to what one gets in computing a derivative in the sense of distributions: a derivative plus a jump. The similarity is quite natural; here also, we have a derivative inside a duality.

Let us now return to the proof of Theorem 12 .

Using Lemma 13, we find that

$$
\begin{aligned}
& {[P Q, R S]=\frac{1}{p+q} \sum_{i_{1}, \ldots, i_{p}} \sum_{j_{2}, \ldots, j_{r}} c_{i_{1}, \ldots, i_{p}} \bar{d}_{i_{1}, j_{2}, \ldots, i_{r}}\left[x_{i_{2}} \cdots x_{i_{p}} Q, x_{j_{2}} \cdots x_{j_{r}} S\right]} \\
& \quad+\frac{1}{p+q} \sum_{i_{1}, \ldots, i_{p}} \sum_{j_{1}, \ldots, j_{r}} c_{i_{1}, \ldots, i_{p}} \bar{d}_{j_{1}, \ldots, j_{r}}\left[x_{i_{2}} \cdots x_{i_{p}} Q, x_{j_{1}} \frac{\partial}{\partial x_{i_{1}}}\left(x_{j_{2}} \cdots x_{j_{r}} S\right)\right]
\end{aligned}
$$

Repeating the process, we get

$$
\begin{aligned}
= & \frac{r}{p+q} \sum_{i_{1}, \ldots, i_{p}} \sum_{j_{2}, \ldots, j_{r}} c_{i_{1}, \ldots, i_{p}} \bar{d}_{i_{1}, j_{2}, \ldots, j_{r}}\left[x_{i_{2}} \cdots x_{i_{p}} Q, x_{j_{2}} \cdots x_{j_{r}} S\right] \\
& +\frac{1}{p+q} \sum_{i_{1}, \ldots, i_{p}} \sum_{j_{1}, \ldots, j_{r}} c_{i_{1}, \ldots, i_{p}} \bar{d}_{j_{1}, \ldots, j_{r}}\left[x_{i_{2}} \cdots x_{i_{p}} Q, x_{j_{1}} \cdots x_{j_{r}} S_{i_{1}}\right]
\end{aligned}
$$


In order to argue by induction, we define

$$
\begin{aligned}
L(l, k)=\sum_{i_{1}, \ldots, i_{p}} \sum_{j_{l+1}, \ldots, j_{r}} c_{i_{1}, \ldots, i_{p}} \bar{d}_{i_{1}, \ldots, i_{l}, j_{l+1}, \ldots, j_{r}} \\
\quad \times\left[x_{i_{p-k+1}} \cdots x_{i_{p}} Q, x_{j_{l+1}} \cdots x_{j_{r}} S_{i_{l+1}, \ldots, i_{p-k}}\right] .
\end{aligned}
$$

In this notation, $l$ stands for the numbers of "links" between the $c$ 's and the $d$ 's (that is, the number of indices appearing in both) and $k$ stands for the number of variables before $Q$ (from $x_{i_{p-k+1}}$ to $x_{i_{p}}$ ). We observe that $[P Q, R S]=L(0, p)$.

Then the same computation as above, using Lemma 13, yields the induction formula:

$$
L(l, k)=\frac{r-l}{q+k} L(l+1, k-1)+\frac{1}{k+q} L(l, k-1) .
$$

From this formula, we deduce by induction on $j$ that, for all $j$,

$$
\begin{aligned}
L(0, p)=\frac{(p+q-j) !}{(p+q) !}( & r(r-1) \cdots(r-j+1) L(j, p-j) \\
& +\left(\begin{array}{c}
j \\
1
\end{array}\right) r(r-1) \cdots(r-j+2) L(j-1, p-j) \\
& \vdots \\
& +\left(\begin{array}{c}
j \\
\lambda
\end{array}\right) r \cdots(r-j+\lambda+1) L(j-\lambda, p-j) \\
& \vdots \\
& \left.+\left(\begin{array}{c}
j \\
j-1
\end{array}\right) r L(1, p-j)+\left(\begin{array}{l}
j \\
j
\end{array}\right) L(0, p-j)\right)
\end{aligned}
$$

Taking $j=p$, we get

$$
\begin{aligned}
L(0, p)=\frac{q !}{(p+q) !} & \left(r(r-1) \cdots(r-p+1) L(p, 0)+\cdots+\left(\begin{array}{l}
p \\
l
\end{array}\right) r \cdots\right. \\
& \left.(r-l+1) L(l, 0)+\cdots+\left(\begin{array}{l}
p \\
1
\end{array}\right) r L(1,0)+L(0,0)\right) .
\end{aligned}
$$

But, for every $l$,

$$
L(l, 0)=\sum_{i_{1}, \ldots, i_{p}} \sum_{j_{l+1}, \ldots, j_{r}} c_{i_{1}, \ldots, i_{p}} \bar{d}_{i_{1}, \ldots, i_{l}, j_{l+1}, \ldots, j_{r}}\left[Q, x_{j_{l+1}} \cdots x_{j_{r}} S_{i_{l+1}, \ldots, i_{p}}\right]
$$

and by Lemma 9

$$
\begin{aligned}
& =\frac{(q-r+l) !}{q !} \sum_{i_{1}, \ldots, i_{p}} \sum_{j_{l+1}, \ldots, j_{r}} c_{i_{1}, \ldots, i_{p}} \bar{d}_{i_{1}, \ldots, i_{l}, j_{l+1}, \ldots, j_{r}}\left[Q_{j_{l+1} \ldots j_{r}}, S_{i_{l+1}, \ldots, i_{p}}\right] \\
& =\frac{(q-r+l) !}{q !} \sum_{i_{1}, \ldots, i_{l}}\left[\sum_{j_{l+1}, \ldots, j_{r}} \bar{d}_{i_{1}, \ldots, i_{l}, j_{l+1}, \ldots, j_{r}} Q_{j_{l+1}, \ldots, j_{r}}, \sum_{i_{l+1}, \ldots, i_{p}} \bar{c}_{i_{1}, \ldots, i_{p}} S_{i_{l+1}, \ldots, i_{p}}\right] \\
& =\frac{(q-r+l) !}{q !} \frac{(r-l) !}{r !} \frac{(p-l) !}{p !} \sum_{i_{1}, \ldots, i_{l}}\left[R_{i_{1}, \ldots, i_{l}}^{*}(D) Q, P_{i_{1}, \ldots, i_{l}}^{*}(D) S\right] .
\end{aligned}
$$


Substituting each $L(l, 0)$ into $L(0, p)$ above yields the announced result.

Second proof. Using Euler's formula, we can write $P=\frac{1}{p} \sum x_{i} P_{i}$, and thus

$$
[P Q, R S]=\frac{1}{p} \sum_{i}\left[x_{i} P_{i} Q, R S\right]=\frac{1}{p(r+s)} \sum_{i}\left[P_{i} Q,(R S)_{i}\right]
$$

Repeating this process on $P_{i}$, we get

$$
[P Q, R S]=\frac{(r+s-p) !}{p !(r+s) !} \sum_{i_{1}, \ldots, i_{p}}\left[P_{i_{1}, \ldots, i_{p}} Q,(R S)_{i_{1}, \ldots, i_{p}}\right]
$$

We now study the derivative $(R S)_{i_{1}, \ldots, i_{p}}$. Let $\tau_{p}$ be the set of partitions of $\{1, \ldots, p\}$ into two subsets. If $u, v$ are two such subsets, we let $k$ be the number of elements in $u, k^{\prime}$ the number of elements in $v$ (of course $k+k^{\prime}=$ $p)$. We also write $u(j)$ for the $j$ th element of $u, v\left(j^{\prime}\right)$ for the $j^{\prime}$ th element of $v$. Then

$$
(R S)_{i_{1}, \ldots, i_{p}}=\sum_{(u, v) \in \tau_{p}} R_{i_{u(1)}, \ldots, i_{u(k)}} S_{i_{v(1)}, \ldots, i_{v\left(k^{\prime}\right)}}
$$

Therefore

$$
[P Q, R S]=\frac{q !}{p !(p+q) !} \sum_{i_{1}, \ldots, i_{p}} \sum_{(u, v) \in \tau_{p}}\left[P_{i_{1}, \ldots, i_{p}} Q, R_{i_{u(1)}, \ldots, i_{u(k)}} S_{i_{v(1)}, \ldots, i_{v\left(k^{\prime}\right)}}\right]
$$

Repeating the same argument, we get

$$
\begin{aligned}
{[P Q, R S]=} & \frac{q !}{p !(p+q) !} \sum_{(u, v) \in \tau_{p}} \sum_{i_{1}, \ldots, i_{p}} \frac{(q-r+k) !}{(r-k) ! q !} \\
& \times \sum_{j_{k+1}, \ldots, j_{r}}\left[\left(P_{i_{1}, \ldots, i_{p}} Q\right)_{j_{k+1}, \ldots, j_{r}}, R_{i_{u(1)}, \ldots, i_{u(k)} j_{k+1}, \ldots, j_{r}} S_{\left.i_{v(1)}, \ldots, i_{v\left(k^{\prime}\right)}\right]}\right] .
\end{aligned}
$$

But $P_{i_{p}, \ldots, i_{p}}$ is a scalar, and therefore

$$
\begin{aligned}
{[P Q, R S]=} & \frac{q !}{p !(p+q) !} \sum_{(u, v) \in \tau_{p}} \sum_{i_{1}, \ldots, i_{p}} \frac{(q-r+k) !}{(r-k) ! q !} \\
& \times \sum_{j_{k+1}, \ldots, j_{r}}\left[P_{i_{1}, \ldots, i_{p}} Q_{j_{k+1}, \ldots, j_{r}}, R_{i_{u(1)}, \ldots, i_{u(k)} j_{k+1}, \ldots, j_{r}} S_{\left.i_{v(1)}, \ldots, i_{v\left(k^{\prime}\right)}\right]}\right.
\end{aligned}
$$


Since both $P_{i_{1}, \ldots, i_{p}}$ and $R_{i_{u(1)}, \ldots, i_{u(k)}, j_{k+1}, \ldots, j_{r}}$ are scalars, this is also

$$
\begin{aligned}
& =\frac{1}{p !(p+q) !} \sum_{(u, v) \in \tau_{p}} \sum_{i_{1}, \ldots, i_{p}} \frac{(q-r+k) !}{(r-k) !} \\
& \times \sum_{j_{k+1}, \ldots, j_{r}}\left[\bar{R}_{i_{u(1)}, \ldots, i_{u(k)}, j_{k+1}, \ldots, j_{r}} Q_{j_{k+1}, \ldots, j_{r}}, \bar{P}_{i_{1}, \ldots, i_{p}} S_{i_{v(1)}, \ldots, i_{v\left(k^{\prime}\right)}}\right] \\
& =\frac{1}{p !(p+q) !} \sum_{(u, v) \in \tau_{p}} \frac{(q-r+k) !}{(r-k) !} \sum_{i_{u(1)}, \ldots, i_{u(k)}} \\
& \times\left[\sum_{j_{k+1}, \ldots, j_{r}} \bar{R}_{i_{u(1)}, \ldots, i_{u(k)}, j_{k+1}, \ldots, j_{r}} Q_{j_{k+1}, \ldots, j_{r}}, \sum_{i_{v(1)}, \ldots, i_{v\left(k^{\prime}\right)}} \bar{P}_{i_{1}, \ldots, i_{p}} S_{i_{v(1)}, \ldots, i_{v\left(k^{\prime}\right)}}\right] \\
& =\frac{1}{p !(p+q) !} \sum_{(u, v) \in \tau_{p}} \frac{(q-r+k) !}{(r-k) !} \\
& \times \sum_{i_{u(1)}, \ldots, i_{u(k)}}\left[(r-k) ! R_{i_{u(1)}, \ldots, i_{u(k)}}^{*}(D) Q,(p-k) ! P_{i_{u(1)}, \ldots, i_{u(k)}}^{*}(D) S\right] \\
& =\frac{1}{p !(p+q) !} \sum_{(u, v) \in \tau_{p}}(q-r+k) !(p-k) ! \sum_{i_{1}, \ldots, i_{k}}\left[R_{i_{1}, \ldots, i_{k}}^{*}(D) Q, P_{i_{1}, \ldots, i_{k}}^{*}(D) S\right] \\
& =\frac{1}{p !(p+q) !} \sum_{k=0}^{p}\left(\begin{array}{l}
p \\
k
\end{array}\right)(q-r+k) !(p-k) ! \sum_{i_{1}, \ldots, i_{k}}\left[R_{i_{1}, \ldots, i_{k}}^{*}(D) Q, P_{i_{1}, \ldots, i_{k}}^{*}(D) S\right],
\end{aligned}
$$

which gives

$$
[P Q, R S]=\frac{1}{(p+q) !} \sum_{k=0}^{p} \frac{(q-r+k) !}{k !} \sum_{i_{1}, \ldots, i_{k}}\left[R_{i_{1}, \ldots, i_{k}}^{*}(D) Q, P_{i_{1}, \ldots, i_{k}}^{*}(D) S\right]
$$

and concludes the second proof.

Taking $P=R, Q=S$ (thus $q=s, p=r$ ), we immediately deduce

Corollary 14. For any homogeneous polynomials $P, Q$ of degrees $p$ and $q$ respectively,

$$
[P Q]^{2}=\frac{1}{(p+q) !} \sum_{k=0}^{p} \frac{(q-p+k) !}{k !} \sum_{i_{1}, \ldots, i_{k}}\left[P_{i_{1}}^{*}, \ldots, i_{k}(D) Q\right]^{2} .
$$

Since all terms on the right-hand side are positive, we deduce, taking $k=p$,

$$
[P Q]^{2} \geq \frac{q !}{(p+q) ! p !} \sum_{i_{1}, \ldots, i_{p}}\left[P_{i_{1}, \ldots, i_{p}}^{*}(D) Q\right]^{2} .
$$

But $P_{i_{1}, \ldots, i_{p}}^{*}=p ! \bar{c}_{i_{1}}, \ldots, i_{p}$ is just a constant. So

$$
\sum_{i_{1}, \ldots, i_{p}}\left[P_{i_{1}, \ldots, i_{p}}^{*}(D) Q\right]^{2}=p !^{2} \sum_{i_{1}, \ldots, i_{p}}\left|c_{i_{1}, \ldots, i_{p}}\right|^{2}[Q]^{2}=p !^{2}[P]^{2}[Q]^{2},
$$

and we deduce that

$$
[P Q]^{2} \geq \frac{p ! q !}{(p+q) !}[P]^{2}[Q]^{2}
$$

which is Bombieri's inequality [4]. 
Using Bombieri's proof in [4], J. L. Frot noted that the quantity $\sum_{i}\left[P_{i} Q, P Q_{i}\right]$ was real and positive. We deduce a stronger statement from Theorem 12:

Theorem 15. For all homogeneous polynomials $P, Q$, with degree $m$ and $n$ respectively, we have

$\sum\left[P_{i} Q, P Q_{i}\right]=\frac{1}{(m+n-1) !} \sum_{k=0}^{m} \frac{(n-m+k) !}{k !}(m-k) \sum_{i_{1}, \ldots, i_{k}=1}^{N}\left[P_{i_{1}, \ldots, i_{k}}^{*}(D) Q\right]^{2}$.

Proof. We set $\varphi(P, Q)=\sum\left[P_{i} Q, P Q_{i}\right]$. Then an immediate computation shows that

$$
\varphi(P, Q)=m(m+n)[P Q]^{2}-\sum_{i=1}^{N}\left[P_{i} Q\right]^{2} .
$$

Applying Theorem 11, we have

$$
[P Q]^{2}=\frac{1}{(m+n) !} \sum_{k=0}^{m} \frac{(n-m+k) !}{k !} \sum_{i_{1}, \ldots, i_{k}=1}^{N}\left[P_{i_{1}, \ldots, i_{k}}^{*}(D) Q\right]^{2},
$$

but

$$
\left(P_{i}\right)_{i_{1}, \ldots, i_{k-1}}(D)=P_{i_{1}, \ldots, i_{k-1}, i}(D),
$$

and thus

$$
\begin{aligned}
\varphi(P, Q)= & \frac{m}{(m+n-1) !} \sum_{k=0}^{m} \frac{(n-m+k) !}{k !} \sum_{i_{1}, \ldots, i_{k}}\left[P_{i_{1}, \ldots, i_{k}}^{*}(D) Q\right]^{2} \\
& -\sum_{k=1}^{m} \frac{(n-m+k) !}{(k-1) !} \sum_{i, i_{1}, \ldots, i_{k-1}}\left[P_{i_{1}, \ldots, i_{k-1}, i}^{*}(D) Q\right]^{2},
\end{aligned}
$$

which gives the result.

The previous results are algebraic identities. They have analytic consequences, which are quantitative estimates, obtained by means of inequalities. These estimates will be the object of forthcoming papers.

Added in proof. As pointed out by $\mathrm{D}$. Zielberger, a verification of the identity in Theorem 12 can be obtained, taking for $P, Q, R, S$ monomials and computing $P_{i_{1}, \ldots, i_{k}}^{*}(D) S$ and $R_{i_{1}, \ldots, i_{k}}^{*}(D) Q$. The result then can be derived from Chu's identity $\sum_{i \geq 0}\left(\begin{array}{c}r \\ i\end{array}\right)\left(\begin{array}{c}s \\ p-i\end{array}\right)=\left(\begin{array}{c}r+s \\ p\end{array}\right)$.

\section{REFERENCES}

1. R. Aron and J. Globevnik, Interpolation by analytic functions on $c_{0}$, Math. Proc. Cambridge Philos. Soc. 104 (1988), 295-302.

2. B. Beauzamy, Introduction to Banach spaces and their geometry, 2nd ed., North-Holland, Amsterdan, 1985.

3. __ Products of many-variable polynomials: pairs that are maximal in Bombieri's norm, J. Number Theory (to appear).

4. B. Beauzamy et al., Products of polynomials in many variables, J. Number Theory 36 (1990), 219-245.

5. B. Beauzamy, J. L. Frot, and C. Millour, Massively parallel computations on many-variable polynomials: when seconds count, Ann. Math. Artificial Intelligence, Special Vol.: Mathematics and Computer Science (M. Nivat and S. Grigorieff, eds.) (to appear). 
6. S. Dineen, Complex analysis in locally convex spaces, North-Holland, Amsterdam, 1981.

7. L. Harris, Bounds on the derivatives of holomorphic functions of vectors, Analyse Fonctionnelle et Applications (Colloque, Rio de Janeiro, 1972; L. Nachbin, ed.), Actualités Sci. Indust., no. 1367, Hermann, Paris, 1975, pp. 145-163.

8. Y. Legrandgérard, On some representations of the norm $[\cdot]_{2}$, manuscript.

9. M. Marden, Geometry of polynomials, 3rd ed., Amer. Math. Soc., Providence, RI, 1985.

10. B. Reznick, An inequality for products of polynomials, Proc. Amer. Math. Soc. 117 (1993), 1063-1073.

11. Y. Sarantopoulos, Extremal multilinear forms on Banach spaces, Proc. Amer. Math. Soc. 99 (1987). 340-346.

12. I. Zalduendo, An estimate for multilinear forms on $l^{p}$ spaces, Proc. Roy. Irish Acad. Sect. A 93 (1993), 137-142.

Institut de Calcul Mathématique, 37, rue Tournefort, 75005 Paris, France 